\title{
Intravascular ultrasound in percutanous coronary intervention for chronic total occlusions: predictors of use, indications and in-hospital outcome
}

\author{
Boukhris $\mathrm{M}^{1,2}$, Seminara $\mathrm{S}^{1}$, Elleuche $\mathrm{A}^{2}$, Krarti $\mathrm{T}^{2}$, Abdelbasset $\mathrm{HM}^{3}$, Tomasello $\mathrm{SD}^{1}$ and Galassi $\mathrm{AR}^{1,4 *}$ \\ ${ }^{1}$ Department of Clinical and Experimental Medicine, University of Catania, Italy \\ ${ }^{2}$ Department of Cardiology, Abderrahmen Mami Hospital, Ariana, Faculty of Medicine of Tunis, University of Tunis El Manar, Tunisia \\ ${ }^{3}$ Maadi Military Hospital, Military Medical Academy, Cairo \\ ${ }^{4}$ University Heart Center, University Hospital Zurich Switzerland
}

\begin{abstract}
Background: Intravascular ultrasound (IVUS) has various potential roles in percutaneous coronary intervention (PCI) for chronic total occlusions (CTOs). The use of IVUS in CTO PCI attempts has increased during the last years.

Aim: We sought to describe the experience of a CTO dedicated operator with IVUS in CTO PCI identifying the independent predictors of the need of such a device and assessing the immediate outcome of IVUS guided CTO PCI.

Method: We conducted a retrospective analysis of 593 patients (mean age $63.2 \pm 10.1$ years, $90.9 \%$ men) who underwent 601 CTO PCI attempts by a single CTOdedicated operator from January 2013 to December 2016. Stepwise logistic regression analysis was performed to identify the independent predictors of IVUS use. Procedural details and in-hospital outcome were compared between the IVUS and non IVUS guided CTO PCI attempts.

Result: IVUS guidance was required in 52 CTO PCI procedures (8.6\%). At multivariate analysis, the independent predictors of IVUS use in CTO PCI were the following: blunt stump ([odds ratio] OR=3.1 95\% CI 1.73-5.2; $\mathrm{p}=0.001$ ), and ostial occlusion (OR=1.93 95\% CI 1.26 $-3.9 ; \mathrm{p}=0.013$ ). Similar technical (94.2 \% vs. $92 \% ; \mathrm{p}=0.783)$ and clinical success rates $(90.4 \%$ vs. $89.9 \%$; $\mathrm{p}=0.871)$ were observed between IVUS and non-IVUS guided CTO PCI. Lower contrast load was required in IVUS guided CTO PCI attempts $(359.4 \pm 203.6 \mathrm{ml}$ vs. $431.6 \pm 212.9 \mathrm{ml} ; \mathrm{p}=0.002)$.
\end{abstract}

Conclusion: IVUS guided CTO PCI attempts are efficient and safe procedures and might reduce the need for contrast load.

\begin{abstract}
Abbreviations: CTO: chronic total occlusion; ERCTO: European registry of chronic total occlusions; IVUS: intravascular ultrasound; JCTO: Japanese multicenter CTO; MACE: major adverse cardiac events; MI: myocardial infarction; PCI: percutaneous coronary intervention

\section{Introduction}

Intravascular ultrasound (IVUS), a catheter based imaging technique providing high resolution cross-sectional images of the lumen and vessel wall, has been used for more than two decades to guide PCI in different subsets of coronary artery lesions in diseased patent coronary arteries [1-3]. Recently, the interest in IVUS use during percutaneous coronary intervention (PCI) of chronic total occlusions (CTOs) has dramatically increased [4-6]. Indeed, IVUS has various potential roles in the recanalization of CTOs, some specific to wire crossing, others in common with other complex long calcified lesions such as optimal sizing and expansion of the stents [7]. The use of IVUS among European Registry of Chronic Total Occlusions (ERCTO) has increased during the last years. However, predictors of employing such an imaging modality in treating CTO patients are still scarcely identified. In addition, the impact of IVUS guided CTO on immediate outcome needs to be further investigated.
\end{abstract}

In the current study, we aimed to describe the experience of a CTO dedicated operator with IVUS in CTO PCI identifying the independent predictors of the need of such a device and assessing the immediate outcome of IVUS guided CTO PCI.

\section{Methods}

\section{Study population}

We conducted a retrospective analysis of consecutive patients with at least 1 native coronary artery CTO subjected to percutaneous recanalization attempts performed by a single expert interventional cardiologist (A.R.G) from January 2013 to December 2016 inclusive. All procedures were scheduled (not ad hoc $\mathrm{PCI}$ ), and patients were selected on the basis of the presence of symptoms, viability of the myocardium subtended by the CTO artery, and inducible ischemia $(>10 \%)$ in the CTO artery territory, as demonstrated by functional imaging tests. In patients with more than $1 \mathrm{CTO}$, only 1 CTO vessel was attempted per procedure. All CTO lesions were attempted only once.

Correspondence to: Alfredo R. Galassi, Via Antonello da Messina 75, Acicastello, 95021 Catania, Italy, Tel: +39-095-7263122; Fax: +39-095-7263124; E-mail: argalassi@gmail.com

Key words: chronic total occlusions; IVUS; blunt stump; proximal cap

Received: October 02, 2017; Accepted: October 27, 2017; Published: October 31, 2017 
Generally, CTO PCI attempt was performed via a double-femoral $7 \mathrm{Fr}$ access, and dual injections were used in presence of contralateral collaterals. The sequence of use of wiring techniques and the guidewire selection were left entirely to the operator's discretion.

The use of IVUS was also left to the operator's discretion. In all IVUS guided CTO PCI, regular Eagle Eye IVUS imaging probe (Phillips Volcano, Nederlands) was employed.

\section{Definitions}

Coronary CTOs were defined as angiographic evidence of total occlusions with TIMI (Thrombolysis In Myocardial Infarction) flow grade 0 and estimated durations of at least 3 months [8]. Visible calcifications and vessel tortuosity were defined as previously described [9]. Collateral filling of the CTO artery from other patent vessels was graded using the Rentrop classification [10]. To assess the difficulty of attempted CTO lesions and procedures, Japanese Multicenter CTO CTO (J-CTO) score and ORA score were calculated as described by Morino et al. [9] and Galassi et al. [11], respectively.

Technical success was defined as angiographic success (final residual stenosis $<20 \%$ by visual estimation and TIMI flow grade 3 after CTO recanalization). Clinical success was defined as angiographic success with no in-hospital major adverse cardiac events (MACE) or contrast-induced nephropathy.In-hospital MACE included cardiac death, Q-wave and non-Q-wave myocardial infarction (MI), tamponade, recurrent symptoms requiring urgent repeat target vessel revascularization (with either PCI or coronary artery bypass grafting).

In all patients, creatine kinase-MB was evaluated $6 \mathrm{~h}$ after the procedure and until normalization if the levels were abnormal. Non-Qwave $\mathrm{MI}$ was defined as creatine kinase-MB enzyme elevation $>3$ times the upper limit of normal. Contrast-induced nephropathy was defined as an increase of $25 \%$ or $0.5 \mathrm{mg} / \mathrm{dl}$ in serum creatinine level at 24 to 48 $\mathrm{h}$ after PCI in comparison with baseline values [12].

\section{Statistical analysis}

Clinical characteristics, angiographic features, and in-hospital events were reported through standard descriptive analyses for large samples assumed to follow a normal distribution. Continuous variables are presented as mean \pm SD and categorical variables as frequencies and percentages. Student $t$ tests and chi-square tests (or Fisher exact tests when at least $25 \%$ of values showed expected cell frequencies $<5$ ) were used for comparison between the 2 groups. We tried to identify clinical and/or angiographic factors predicting the success the use of IVUS in CTO PCI procedures through stepwise logistic regression analysis. Univariate analysis was initially performed; univariate variables with $\mathrm{p}$ values $<0.10$ were thereafter included in the multivariate model. A $\mathrm{p}$ value $<0.05$ was considered to indicate statistical significance. All data were processed using SPSS version 21.0 (SPSS, Chicago, Illinois).

\section{Result}

From January 2013 to December 2016, 593 patients (mean age 63.2 \pm 10.1 years, $90.9 \%$ men) underwent 601 CTO PCI attempts by a single CTO-dedicated operator. IVUS guidance was required in 52 CTO PCI procedures $(8.6 \%)$; and the use of IVUS has significantly increased from $4.3 \%$ to $11.5 \%$ between 2013 and $2016(\mathrm{p}=0.03$ ) (Figure 1).

No difference in terms of clinical characteristics was observed between patients who underwent IVUS guided CTO PCI and those in whom only angiography guided CTO PCI attempt was performed (Table 1). IVUS guidance was required in presence of blunt stamp,
Table 1. Baseline clinical characteristics of the study population

\begin{tabular}{|l|c|c|c|c|}
\hline & $\begin{array}{c}\text { Overall } \\
\mathbf{( N = 5 9 3 )}\end{array}$ & $\begin{array}{c}\text { IVUS guided } \\
\text { CTO PCI } \\
\mathbf{( N = 5 2 )}\end{array}$ & $\begin{array}{c}\text { Non IVUS } \\
\text { guided CTO } \\
\text { PCI (N=541) }\end{array}$ & P value \\
\hline Age, years, mean \pm SD & $63.2 \pm 10.1$ & $64.1 \pm 9.6$ & $63 \pm 11.8$ & 0.921 \\
\hline Males, n (\%) & $539(90.9)$ & $47(90.4)$ & $493(91.1)$ & 0.799 \\
\hline Diabetes mellitus, n (\%) & $180(30.4)$ & $16(30.7)$ & $164(30.3)$ & 0.903 \\
\hline Hypertension, n (\%) & $427(72)$ & $36(67.3)$ & $391(72.3)$ & 0.421 \\
\hline Hyperlipidemia, n (\%) & $381(64.2)$ & $35(67.3)$ & $346(63.9)$ & 0.731 \\
\hline CKD, n (\%) & $65(11)$ & $6(11.3)$ & $59(10.9)$ & 0.931 \\
\hline Smokers , n (\%) & $304(51.3)$ & $26(50)$ & $278(51.4)$ & 0.613 \\
\hline Prior MI, n (\%) & $252(42.5)$ & $24(46.1)$ & $228(42.1)$ & 0.490 \\
\hline LVEF & $50.2 \pm 7.1$ & $51.1 \pm 5.9$ & $50 \pm 8.2$ & 0.772 \\
\hline Prior revascularization & & & & 0.572 \\
\hline PCI, n (\%) & $304(51.3)$ & $24(46.1)$ & $280(51.7)$ & \\
\hline CABG, n (\%) & $42(7.1)$ & $4(7.7)$ & $38(7)$ & \\
\hline Both, n (\%) & $31(5.2)$ & $2(3.8)$ & $29(5.3)$ & \\
\hline Multi-vessel CAD, n (\%) & $430(72.5)$ & $37(71.6)$ & $393(72.6)$ & 0.897 \\
\hline
\end{tabular}

Abbreviations: $\mathrm{CABG}=$ coronary artery bypass grafting; $\mathrm{CAD}=$ coronary artery disease $\mathrm{CTO}=$ chronic total occlusion; IVUS=intravascular ultrasound; LVEF=left ventricular ejection fraction; $\mathrm{MI}=$ myocardial infarction; $\mathrm{PCI}=$ percutaneous coronary intervention.

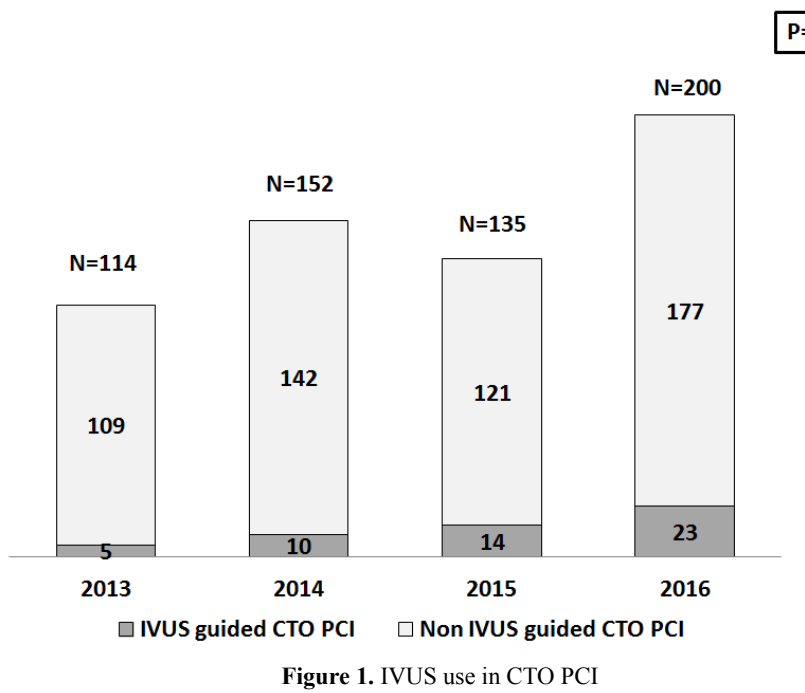

$\mathrm{CTO}=$ chronic total occlusion; IVUS=intravascular ultrasound; $\mathrm{PCI}=$ percutaneous coronary intervention.

more in ostial CTO, in presence of side branch at proximal cap, in CTO lesions priorly attempted and in case of bad distal vessel filling from collaterals (Table 2). At multivariate analysis, the independent predictors of IVUS use in CTO PCI were the following: blunt stump $(\mathrm{OR}=3.195 \% \mathrm{CI} 1.73-5.2 ; \mathrm{p}=0.001)$, and ostial occlusion $(\mathrm{OR}=1.93$ 95\% CI 1.26 -3.9; $\mathrm{p}=0.013$ ) (Figure 2).

Overall, retrograde approach was used in 229 procedures (38.1\%). IVUS was more often used in antegrade approach than in retrograde approach $(76.9 \%$ vs. $23.1 \%, \mathrm{p}=0.03)$. The two major indications were antegrade proximal cap puncture and stent optimization. Figure 3 illustrated the different indications for IVUS use in both antegrade and retrograde approach.

Similar technical ( $94.2 \%$ vs. $92 \% ; \mathrm{p}=0.783)$ and clinical success rates $(90.4 \%$ vs. $89.9 \%$; $\mathrm{p}=0.871)$ were observed between IVUS and non-IVUS guided CTO PCI. Procedural and fluoroscopy times were similar between the two groups, while lower contrast load was required in IVUS guided CTO PCI attempts $(359.4 \pm 203.6 \mathrm{ml} v s .431 .6 \pm 212.9$ $\mathrm{ml} ; \mathrm{p}=0.002$ ) (Figure 3). 
Table 2. Baseline angiographic characteristics

\begin{tabular}{|l|c|c|c|c|}
\hline & $\begin{array}{c}\text { Overall } \\
\mathbf{( N = 6 0 1 )}\end{array}$ & $\begin{array}{c}\text { IVUS guided } \\
\text { CTO PCI } \\
\mathbf{( N = 5 2 )}\end{array}$ & $\begin{array}{c}\text { Non IVUS } \\
\text { guided CTO } \\
\text { PCI (N=549) }\end{array}$ & P value \\
\hline CTO target vessel & & & & 0.714 \\
\hline LAD, n (\%) & $185(30.8)$ & $15(28.8)$ & $170(31)$ & \\
\hline LCx, n (\%) & $106(17.6)$ & $10(19.2)$ & $96(17.5)$ & \\
\hline RCA, n (\%) & $310(51.6)$ & $27(51.9)$ & $283(51.5)$ & \\
\hline CTO length $\geq \mathbf{2 0 m m , ~ n ~ ( \% ) ~}$ & $465(77.4)$ & $40(76.2)$ & $425(77.4)$ & 0.867 \\
\hline Ostial location, n (\%) & $105(17.5)$ & $20(38.5)$ & $85(15.5)$ & $\mathbf{0 . 0 0 3}$ \\
\hline In-stent occlusion, n (\%) & $35(5.8)$ & $3(5.7)$ & $32(5.8)$ & 0.785 \\
\hline Visible calcifications, n (\%) & $191(31.8)$ & $18(34.6)$ & $173(31.5)$ & 0.621 \\
\hline Blunt stump, n (\%) & $343(57.1)$ & $40(76.9)$ & $303(55.2)$ & $\mathbf{0 . 0 0 1}$ \\
\hline Tortuosity, n (\%) & $256(42.6)$ & $22(42.3)$ & $234(42.7)$ & 0.777 \\
\hline Previous attempt, n (\%) & $144(24)$ & $18(34.6)$ & $126(22.9)$ & $\mathbf{0 . 0 3 6}$ \\
\hline J-CTO score, mean \pm SD & $2.3 \pm 1.2$ & $2.2 \pm 1.2$ & $2.3 \pm 1.1$ & 0.319 \\
\hline ORA score, mean \pm SD & $0.8 \pm 0.4$ & $0.9 \pm 0.5$ & $0.8 \pm 0.4$ & 0.842 \\
\hline
\end{tabular}

Abbreviations : $\mathrm{CTO}=$ chronic total occlusions; IVUS $=$ intravascular ultrasound $\mathrm{J}-\mathrm{CTO}=$ Japanese multicenter; $\mathrm{LAD}=\mathrm{left}$ anterior descending; $\mathrm{LCx}=\mathrm{left}$ circumflex; $\mathrm{LM}=$ left main; $\mathrm{RCA}=$ right coronary artery.

\section{IVUS-guided Only stent \\ reverse CART optimization \\ 2 cases $\quad 5$ cases \\ $(1.7 \%) \quad(4.2 \%)$}
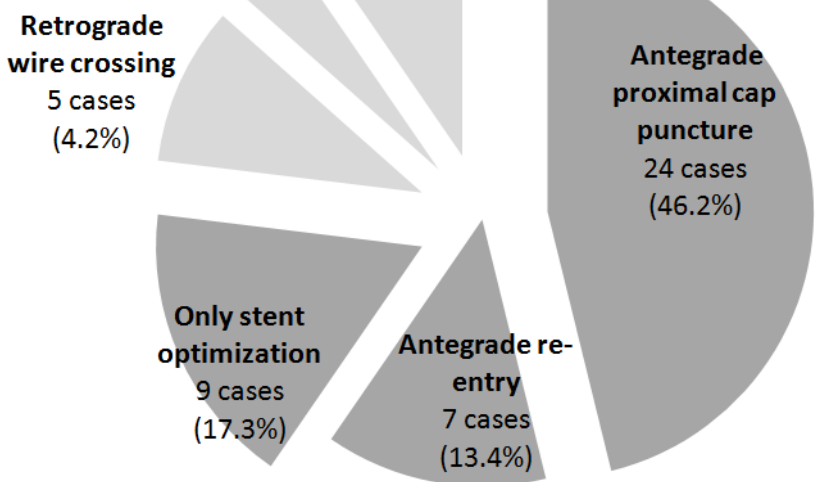

Antegrade approach

Figure 2. Independent predictors of IVUS use in CTO PCI

$\mathrm{CTO}=$ chronic total occlusion; $\mathrm{CI}=$ confidence intervall; IVUS=intravascular ultrasound $\mathrm{J}-\mathrm{CTO}=\mathrm{Japanese}$ multicenter; $\mathrm{OR}=\mathrm{odds}$ ratio; $\mathrm{PCI}=$ percutaneous coronary intervention.

Table 3 summarized the in-hospital outcome of the study population. No significant difference was found in terms of in-hospital MACE occurrence between IVUS and non-IVUS guided CTO PCI attempts $(5.7 \%$ vs. $2.7 \% ; \mathrm{p}=0.29)$. In IVUS guided CTO PCI group, 2 patients $(3.8 \%)$ experienced non-Q wave MI; while a tamponade occurred in a patient $(1.9 \%)$ due to an epicardial collateral rupture. The incidence of contrast induced nephropathy was also similar between the two groups $(1.9 \%$ vs. $2.9 \%$; $\mathrm{p}=0.777)$.

\section{Discussion}

The main findings of the present study can be summarized as follows: i) the use of IVUS in CTO PCI has increased year per year; ii) the independent predictors of IVUS use were ostial location and the presence of blunt stump; iii) the major indications were antegrade proximal cap puncture and stent optimization; iv) IVUS guided CTO
PCI attempts were associated with similar rates of technical and clinical success for CTO percutaneous coronary intervention

Despite the increase in success rates of CTO PCI ( $\triangle 90 \%)$ reported by experienced dedicated operators, the failure of guidewire crossing still represents the commonest reason for failed CTO attempts in presence of certain angiographic features. The added value of IVUS imaging is to provide better understanding of the behavior of the guidewire within the CTO segment; therefore, it could represent a key issue to further increase success rate. In our experience, the use of IVUS has almost tripled from to $4.3 \%$ to $11.5 \%$ in 3 years; however, IVUS guided attempts still represent $1 / 10$ of total CTO PCI procedures. From unpublished data of European CTO Club registry (ERCTO), the percentage of IVUS guided CTO PCI has increased from 5.2\% (5\% in antegrade and 6\% in retrograde) in 2011 to $14.6 \%$ (12.6\% in antegrade and $23.6 \%$ in retrograde). Differently in our study, IVUS was more often used in antegrade procedures.

IVUS has multiple indications in CTO PCI [7]. In antegrade approach, it helps to resolve proximal cap ambiguity, facilitates re-entry into the true lumen after subintimal crossing $[13,14]$ and confirms distal true lumen guidewire position. While, in retrograde CTO attempts,

Table 3. In-hospital clinical outcome

\begin{tabular}{|l|c|c|c|c|}
\hline & $\begin{array}{c}\text { Overall } \\
\text { (N=601) }\end{array}$ & $\begin{array}{c}\text { IVUS } \\
\text { guided } \\
\text { CTO PCI } \\
\text { (N=52) }\end{array}$ & $\begin{array}{c}\text { Non IVUS } \\
\text { guided } \\
\text { CTO PCI } \\
\text { (N=549) }\end{array}$ & P value \\
\hline Cardiac death, n (\%) & $1(0.1)$ & 0 & $1(0.2)$ & 0.714 \\
\hline Q-wave MI, n(\%) & $4(0.7)$ & 0 & $4(0.8)$ & 0.867 \\
\hline Non-Q wave MI, n(\%) & $10(1.7)$ & $2(3.8)$ & $8(1.4)$ & 0.213 \\
\hline $\begin{array}{l}\text { Coronary perforation leading to } \\
\text { tamponade, n(\%) }\end{array}$ & $5(0.8)$ & $1(1.9)$ & $4(0.7)$ & 0.301 \\
\hline $\begin{array}{l}\text { urgent repeat target vessel } \\
\text { revascularization, n(\%) }\end{array}$ & $2(0.3)$ & 0 & $2(0.4)$ & 0.621 \\
\hline In-hospital MACE, n(\%) & $18(3)$ & $3(5.7)$ & $15(2.7)$ & 0.298 \\
\hline Contrast induced nephropathy, n (\%) & $17(2.8)$ & $1(1.9)$ & $16(2.9)$ & 0.777 \\
\hline
\end{tabular}

$\mathrm{CTO}=$ chronic total occlusions; IVUS=intravascular ultrasound; MACE $=$ major adverse cardiac events; $\mathrm{MI}=$ myocardial infarction; $\mathrm{PCI}=$ percutaneous coronary intervention.

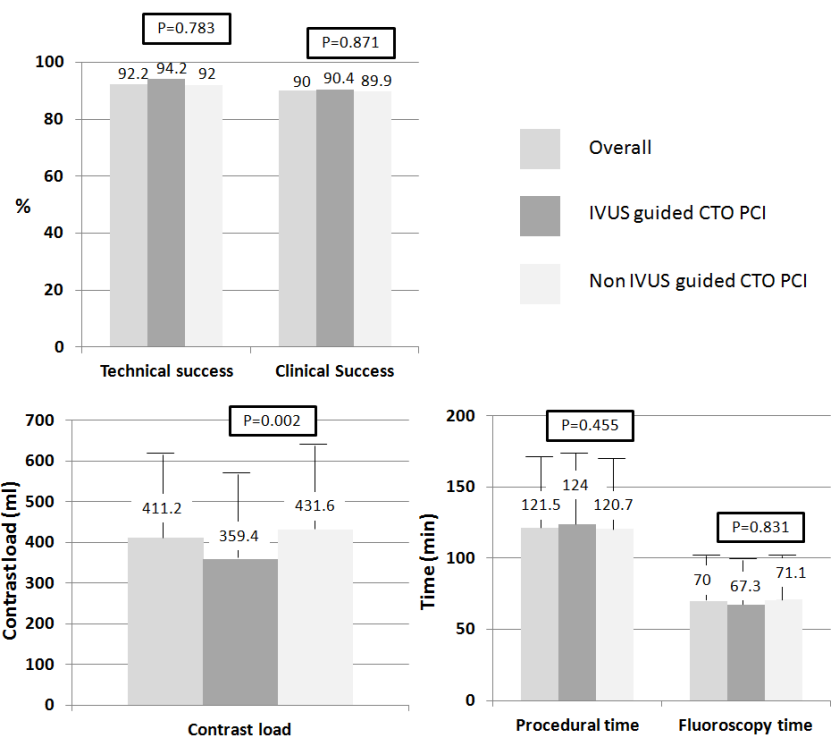

Figure 3. Indications of IVUS in CTO PCI

$\mathrm{CART}=$ controlled antegrade retrograde tracking; $\mathrm{CTO}=$ chronic total occlusion; IVUS=intravascular ultrasound; $\mathrm{PCI}=$ percutaneous coronary intervention. 


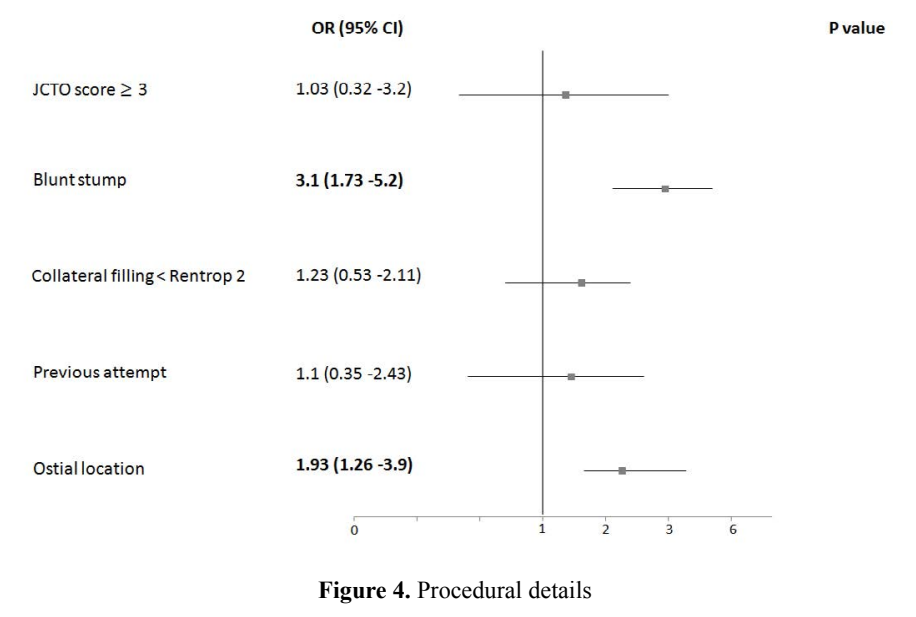

$\mathrm{CTO}=$ chronic total occlusion; IVUS=intravascular ultrasound; $\mathrm{PCI}=$ percutaneous coronary intervention.

IVUS probe (always advanced on an anterograde guidewire) can be used in retrograde guidewire crossing and reverse controlled antegrade retrograde tracking (CART) technique. In addition, after stenting, expansion, apposition, and extension can be further optimized with IVUS assessment [7].

In our study, the presence of blunt stump and an ostial location were demonstrated to independently predict the need for IVUS use during CTO PCI attempts. In a series involving 22 patients who underwent CTO-PCI using IVUS guidance for stumpless ostial CTO, successful outcome was achieved in 17 cases $(77.3 \%)$, while no complications related with the use of IVUS were noticed [15]. Interestingly, When the success rates were analyzed taking into account the date of adoption of this technique, the learning curve had no significant impact on CTO PCI success.

The absence of a well-defined (ambigous) stump is a major limitation for CTO recanalization [16,17]. The major indication of IVUS in our study was antegrade proximal cap puncture. The principle is that the IVUS probe is advanced into a side branch originating at the site of occlusion large enough to accept an IVUS with no extreme angulations or ostial stenosis. During pull-back the precise location of the occlusion and the presence of calcium are evaluated. In our cases, serial examinations with IVUS were performed so that the puncture is done at the site where the angiographic roadmap shows the IVUS transducer when the probe was detected. Once the puncture is performed, subsequent IVUS passes are needed to confirm the intraluminal position of the guidewire for at least the first critical few millimeters. Direct on-line guidance with the probe left at the site can also be an alternative; however, it needs large guiding catheters and IVUS probe could deflect away guidewires and microcatheters from the stump and preclude simultaneous contrast injections. Furthermore, in antegrade approach, the technique of IVUS-guided re-entry can be helpful once the wire is subintimally. Nonetheless, this latter technique requires a large expertise and short-tip Volcano Eagle-Eye (Phillips Volcano, Nederland's) device (placed in subintima) because of the short distance between tip and IVUS sensor.

In case of ostial CTO lesions particularly when a retrograde approach is scheduled, an IVUS probe should be placed (over an antegrade guidewire) immediately in the very ostial segment. In this position, the operator can visualize the exact location of the retrograde guidewire and its re-entry in the true lumen. Without intravascular images, the retrograde guidewire, pushed in the intimal plaque, can be responsible for aortic dissection in case of ostial CTO of the right coronary artery Whereas, in ostial left anterior descending CTO, the retrograde guidewire could be subintimal in the distal predivisional left main and in true lumen in the mid left main. With no IVUS evaluation, externalization of retrograde guidewire and subsequent PCI may lead to left circumflex occlusion [7]. Therefore, in such a situation, thanks to an IVUS probe positioned in the ostial left circumflex, the operator can recognize this dangerous position and modify accordingly the position of retrograde guidewire in left main.

After crossing and predilating a CTO, IVUS provides crucial information about vessel caliber, lesion length, morphology, and landing zone for stents implantation. After stenting, expansion, and apposition can be optimized with IVUS imaging. In the AIR-CTO (Angiographic and clinical comparisons of intravascular ultrasoundversus angiography-guided drug-eluting stent implantation for patients with chronic total occlusion lesions) randomized trial, in comparison with the angiography-guided group, the IVUS-guided group had lower in-stent late lumen loss $(0.28 \pm 0.48 \mathrm{~mm} v s .0 .46 \pm 0.68 \mathrm{~mm} ; \mathrm{p}=0.025)$, and lower restenosis of the "in-true-lumen" stent (3.9\% vs.13.7\%; $\mathrm{p}=0.021)[18]$.

Otherwise, IVUS could be an interesting alterative in theory, when treating CTO patients with chronic kidney disease as it might reduce the need for contrast. However, in two randomized trials, IVUS use was not associated with a reduction of contrast load $[18,19]$. Although in our study, less contrast was required in IVUS guided CTO PCI attempts, this did not result in lower incidence of contrast induced nephropathy.

Regarding clinical outcome, no difference in in-hospital MACE rate was noticed between the two groups in our study. In a randomized trial, Kim et al. [19] compared the mid-term outcome of 201 CTO patients who underwent IVUS-guided versus angiography-guided stenting. At 12 months, the incidence of MACE (composite of cardiac death, MI, or target vessel revascularization) was lower in the IVUS-guided group (2.6\% vs. $7.1 \% ; \mathrm{p}=0.035)$ [19]. However, this latter study was underpowered because of low events rate observed in both groups. In the AIR-CTO trial, there were no differences in the composite MACE and in the individual component of clinical adverse events between the 2 study groups at 1 - and 2-year follow-ups [18]. Therefore, the impact of IVUS guided CTO PCI on clinical outcome is still not yet well established; further studies are needed to better assess it.

\section{Study limitation}

First, its retrospective nature might introduce an inevitable case selection bias. Second, all the procedures were performed by a single CTO-dedicated operator; hence, results might not be applicable to the entire population of CTO operators. Finally, neither mid- nor longterm outcome data were collected.

\section{Conclusion}

In CTO PCI, IVUS might have several potential roles and could be of benefit particularly in the setting of ostial CTO or in presence of ambiguous proximal cap. IVUS guided CTO PCI attempts are efficient and safe procedures and might reduce the need for contrast load.

\section{References}

1. Zhang Y, Farooq V, Garcia-Garcia HM, Bourantas CV, Tian N, et al. (2012) Comparison of intravascular ultrasound versus angiography-guided drug-eluting stent implantation : a meta-analysis of one randomised trial and ten observational studies involving 19,619 patients. EuroIntervention 8: 855-865.[Crossref] 
2. Klersy C, Ferlini M, Raisaro A, Scotti V, Balduini A, et al. (2013) Use of IVUS guided coronary stenting with drug eluting stent: a systematic review and meta-analysis of randomized controlled clinical trials and high quality observational studies. Int $J$ Cardiol 170: 54-63. [Crossref]

3. Ahn JM, Kang SJ, Yoon SH, Park HW, Kang SM, et al. (2014) Meta-analysis of outcomes after intravascular ultrasoundguided versus angiography-guided drug eluting stent implantation in 26,503 patients enrolled in three randomized trials and 14 observational studies. Am J Cardiol. 113: 1338-1347. [Crossref]

4. Tian NL, Gami SK, Ye F, Zhang JJ, Liu ZZ, et al. (2015) Angiographic and clinical comparisons of intravascular ultrasound- versus angiography-guided drug-eluting stent implantation for patients with chronic total occlusion lesions: two-year results from a randomised AIR-CTO study. EuroIntervention 10:1409-1417. [Crossref]

5. Kim BK, Shin DH, Hong MK, Park HS, Rha SW, et al. (2015) Clinical Impact of Intravascular UltrasoundGuided Chronic Total Occlusion Intervention With Zotarolimus-Eluting Versus Biolimus-Eluting Stent Implantation: Randomized Study. Circ Cardiovasc Interv 8: e002592. [Crossref]

6. Karacsonyi J, Alaswad K, Jaffer FA, Yeh RW, Patel M, et al. (2016) Use of Intravascular Imaging During Chronic Total Occlusion Percutaneous Coronary Intervention: Insights from a Contemporary Multicenter Registry. J Am Heart Assoc 5: e003890 [Crossref]

7. Galassi AR, Sumitsuji S, Boukhris M, Brilakis ES, Di Mario C, et al. (2016) Utility of Intravascular Ultrasound in Percutaneous Revascularization of Chronic Total Occlusions: An Overview. JACC Cardiovasc Interv 9: 1979-1991. [Crossref]

8. Sianos G, Barlis P, Di Mario C, Papafaklis MI, Büttner J, et al. (2008) European experience with the retrograde approach for the recanalisation of coronary artery chronic total occlusions. A report on behalf of the EuroCTO club. Eurointervention 4: 84-92. [Crossref]

9. Morino Y, Abe M, Morimoto T, Kimura T, Hayashi Y, et al. (2011) Predicting successful guidewire crossing through chronic total occlusion of native coronary lesions within 30 minutes: the J-CTO (Multicenter CTO Registry in Japan) score as a difficulty grading and time assessment tool. JACC Cardiovasc Interv 4: 213-221. [Crossref]

10. Cohen M, Rentrop KP (1986) Limitation of myocardial ischemia by collatera circulation during sudden controlled coronary artery occlusion in human subjects: a prospective study. Circulation 74: 469-476.

11. Galassi AR, Boukhris M, Azzarelli S, Castaing M, Marzà F, et al. (2016) Percutaneous Coronary Revascularization for Chronic Total Occlusions:A Novel Predictive Score of Technical Failure Using Advanced Technologies. JACC Cardiovasc Interv 9: 911-922. [Crossref]
12. Galassi AR, Tomasello SD, Reifart N, Werner GS, Sianos G, et al. (2011) In-hospital outcomes of percutaneous coronary intervention in patients with chronic total occlusion: insights from the ERCTO (European Registry of Chronic Total Occlusion) registry. Eurointervention 7: 472-479. [Crossref]

13. Werner GS, Diedrich J, Scholz KH, Knies A, Kreuzer H (1997) Vessel reconstruction in total coronary occlusions with a long subintimal wire pathway: use of multiple stents under guidance of intravascular ultrasound. Cathet Cardiovasc Diagn 40: 46-51. [Crossref]

14. Rathore S, Katoh O, Tuschikane E, Oida A, Suzuki T, et al. (2010) A nove modification of the retrograde approach for the recanalization of chronic total occlusion of the coronary arteries intravascular ultrasound-guided reverse controlled antegrade and retrograde tracking. JACC Cardiovasc Interv 3: 155-164. [Crossref]

15. Ryan N, Gonzalo N, Dingli P, Cruz OV, Jiménez-Quevedo P, et al. (2017) Intravascular ultrasound guidance of percutaneous coronary intervention in ostial chronic total occlusions: a description of the technique and procedural results. Int $J$ Cardiovasc Imaging $33: 807-813$. [Crossref]

16. Christopoulos G, Kandzari DE, Yeh RW, Jaffer FA, Karmpaliotis D, et al. (2016) Development and validation of a novel scoring system for predicting technical success of chronic total occlusion percutaneous coronary interventions : the PROGRESS CTO (Prospective Global Registry for the Study of Chronic Total Occlusion Intervention) score. JACC Cardiovasc Interv 9 : 1-9. [Crossref]

17. Alessandrino G, Chevalier B, Lefèvre T, Sanguineti F, Garot P, et al. (2015) A clinical and angiographic scoring system to predict the probability of successful first-attempt percutaneous coronary intervention in patients with total chronic coronary occlusion. JACC Cardiovasc Interv 8:1540-1548. [Crossref]

18. Tian NL, Gami SK, Ye F, Zhang JJ, Liu ZZ, et al. (2015) Angiographic and clinical comparisons of intravascular ultrasound versus angiography-guided drug-eluting stent implantation for patients with chronic total occlusion lesions: two-year results from a randomised AIR-CTO study. EuroIntervention 10: 1409-1417. [Crossref]

19. Kim BK, Shin DH, Hong MK, Park HS, Rha SW, et al. (2015) Clinical impact of intravascular ultrasound-guided chronic total occlusion intervention with zotarolimus eluting versus biolimus-eluting stent implantation: randomized study. Circ Cardiovasc Interv 8: e002592. [Crossref]

Copyright: (C2017 Boukhris M. This is an open-access article distributed under the terms of the Creative Commons Attribution License, which permits unrestricted use, distribution, and reproduction in any medium, provided the original author and source are credited. 